\title{
Erratum: Electron diffraction by plasmon waves [Phys. Rev. B 94, 041404(R) (2016)]
}

\author{
F. J. García de Abajo, B. Barwick, and F. Carbone
}

(Received 2 February 2019; published 14 February 2019)

DOI: 10.1103/PhysRevB.99.079905

The explicit factor of $\gamma$ should be removed from Eqs. (2), (3), and (8) as well as from the displayed equation for $\beta$ on p. 2 of the paper, so they become

$$
\begin{aligned}
\frac{-e v}{\hbar \omega}\left(e^{-i \omega t} \mathcal{E}_{z}-e^{i \omega t} \mathcal{E}_{z}^{*}\right) \phi & =\left(v \frac{\partial}{\partial z}+\frac{\partial}{\partial t}\right) \phi, \\
\frac{\partial f_{\ell}}{\partial z} & =\frac{e}{\hbar \omega}\left(\mathcal{E}_{z}^{*} e^{i \omega z / v} f_{\ell+1}-\mathcal{E}_{z} e^{-i \omega z / v} f_{\ell-1}\right), \\
g & =e \mathcal{E}_{0} \lambda_{\mathrm{p}} / 2 \pi \hbar \omega, \\
\beta(z) & =\frac{e}{\hbar \omega} \int_{-\infty}^{z} d z^{\prime} \mathcal{E}_{z}\left(z^{\prime}\right) e^{-i \omega z^{\prime} / v},
\end{aligned}
$$

respectively. Additionally, the first exponential in Eq. (5) has to be changed, so it becomes

$$
\psi(\mathbf{r}, t)=\frac{1}{4 \pi^{2} \sqrt{V}} \sum_{\ell} e^{i \varphi_{\ell}} \int d^{2} \mathbf{k}_{\perp} e^{i \mathbf{k}_{\ell} \cdot \mathbf{r}} f_{\ell, \mathbf{k}_{\perp}} .
$$

Finally, the calculations presented in Fig. 2 correspond to $g=1$ (upper panels) and $g=5$ (lower panels) instead of $g=1 / 2$ and $g=5 / 2$, respectively. These errors do not affect the main results and conclusions of the paper. 